\title{
Etanercept in the treatment of rheumatoid arthritis
}

\author{
Boulos Haraoui' \\ Vivian Bykerk ${ }^{2}$ \\ 'Clinical Associate Professor of \\ Medicine, University of Montreal, \\ Montreal Canada; ${ }^{2}$ Associate \\ Professor of Medicine, University of \\ Toronto, Toronto Canada
}

\begin{abstract}
Etanercept (ETN) is the first anti-tumor necrosis factor (TNF) agent to be approved for the treatment of rheumatoid arthritis (RA). Over the last 8 years, several clinical trials have shown its efficacy and safety in established and early RA, as well as a monotherapy or in combination with methotrexate. ETN not only reduces the signs and symptoms of RA, but also retards the progression of radiographic damage and improves the quality of life and function of patients. Its safety profile has been predictable since the first clinical trials with no new major safety concerns. Beyond its efficacy in RA, ETN is also indicated for the treatment of psoriatic arthritis. This current report reviews the evidence and the data in RA and psoriatic arthritis (PsA).
\end{abstract}

Keywords: etanercept, tumor necrosis factor- $\alpha$, rheumatoid arthritis, psoriatic arthritis

\section{Introduction}

In the last decade, the treatment of rheumatoid arthritis (RA) has undergone a major revolution. The advent of targeted therapies, mainly against tumor necrosis factoralpha (TNF- $\alpha$ ) and the development of new therapeutic strategies of their early combination with traditional disease modifying anti-rheumatic drugs (DMARD), in particular methotrexate (MTX) and the aim of achieving clinical remission, have dramatically changed the management and hence the prognosis of RA. Etanercept (ETN) was the first biologic response modifier to be approved by the US Food and Drug Administration (FDA) for use in RA. The focus of this current review is the efficacy and safety as well as the current positioning of ETN in the treatment of RA.

\section{Molecular structure and mode of action}

Etanercept is a dimeric human tumor necrosis factor receptor (TNFR) p75-Fc fusion protein made of 2 extra-cellular domains of the human $75 \mathrm{kD}$ (p75) TNFR linked by the constant Fc portion of human immunoglobulin 1 (IgG1). Etanercept is produced by recombinant DNA technology in Chinese hamster ovary $(\mathrm{CHO})$ mammalian cell expression system. It consists of 934 amino acids and has an approximate molecular weight of $150 \mathrm{kD}$.

TNF is a naturally occurring cytokine produced primarily by activated macrophages and $\mathrm{T}$ cells and exists predominantly as a trimer (Beayert and Fiers 1998; Krakauer et al 1999; Locksley et al 2001; McDermott 2001). Two distinct receptors for TNF exist naturally as monomeric molecules on cell surfaces and in soluble forms. One is a $55 \mathrm{kD}$ protein (p55) and the other has a molecular weight of $75 \mathrm{kD}$ (p75). The biological activity of TNF is dependent upon binding to either cell surface TNFR. Monomers of the extracellular portion of the TNFRs naturally cleaved from the cell surface are termed soluble TNF receptors (sTNFR). sTNFRs bind with high affinity to circulating TNF and act as natural antagonists to TNF preventing the TNF molecules from binding to cell-bound receptors.

The dimeric structure of ETN enhances its binding affinity and provides substantially greater competitive inhibition of TNF than monomeric soluble receptors. 
Use of an $\operatorname{IgG} \mathrm{Fc}$ region as a fusion element in this construction imparts a longer serum half-life compared with monomeric soluble receptors.

Etanercept inhibits in vitro the activity of human TNF and is efficacious in many in vivo models of inflammation, including arthritis. Etanercept competitively inhibits the binding of both TNF- $\alpha$ and TNF- $\beta$ (lymphotoxin- $\alpha$ [LT$\alpha]$ ) to cell surface TNF receptors, rendering TNF biologically inactive (Mohler et al 1993).

Etanercept also modulates indirectly different biological responses that are induced or regulated by TNF, such as the expression of adhesion molecules E-selectin and to a lesser extent intercellular adhesion molecule 1 (ICAM-1), the production of interleukin-6 (IL-6) and matrix metalloproteinase 3 (MMP-3) (stromelysin), as well as IL1 (Verschueren et al 1999; Cartina et al 2002).

The immune function of patients with RA who are treated with ETN has been extensively studied (Berg et al 2001; Moreland et al 2002). T-cell responsiveness to microbial antigens as well to collagen type II is not altered. No significant differences are noted between patients treated with ETN or placebo in the phenotypes of peripheral blood leukocytes, T-cell proliferative responses, neutrophil function, delayed type hypersensitivity reactions or serum IgG levels.

\section{Human pharmacokinetics}

The pharmacokinetics of ETN were studied in approximately 300 subjects with doses ranging from $0.125 \mathrm{mg} / \mathrm{m}^{2}$ to $60 \mathrm{mg} / \mathrm{m}^{2}$ administered by a single intravenous (IV) infusion over 30 minutes or by single and multiple subcutaneous (SC) injections. Following a single administration of $25 \mathrm{mg}$ SC to 26 healthy volunteers, the peak serum concentration is reached after a mean of 51 hours with a maximum concentration $\left(\mathrm{C}_{\max }\right)$ of $1.46 \mathrm{mcg} / \mathrm{ml}$ (range 0.37-3.47) (Korth-Bradley et al 2000). The elimination halflife is 68 hours. A twice weekly dosing regimen was devised to maintain a steady-state concentration. Analyses of serum samples from adult RA patients in long-term treatment trials show stable steady-state concentrations of ETN. Recently, a study comparing this traditional dosing regimen with a once weekly injection of $50 \mathrm{mg}$ showed comparable efficacy and pharmacokinetics (Keystone et al 2005). However this study was relatively short; further data using the $50 \mathrm{mg}$ once a week dosing regimen for a longer period of time and assessing radiographic progression are needed.

There is no need for dose adjustment in the presence of renal and/or hepatic impairment. No differences have been observed in pharmacokinetics between men and women. Clearance and volume estimates in patients aged 65 to 87 years are similar to those for patients less than 65 years of age. Concomitant MTX administration does not alter the pharmacokinetics of ETN.

\section{Efficacy of etanercept in rheumatoid arthritis}

Numerous trials have demonstrated the efficacy and safety of ETN in RA. Five major clinical trials are reviewed here. The first randomized phase II trial demonstrating its efficacy in patients with RA was in 1997 (Moreland et al 1997). This was followed by evidence of its efficacy in patients with RA who had suboptimal response to MTX in a 24week, double-blind randomized controlled trial (Weinblatt et al 1999). In this study, 89 patients with persistently active RA despite at least 6 months of MTX therapy on a stable dose of 15-25 mg per week were assigned to receive either ETN $(25 \mathrm{mg}$ ) or placebo SC twice weekly while continuing their stable dose of MTX. The primary measure of clinical response was the American College of Rheumatology criteria for a $20 \%$ improvement in measures of disease activity (ACR20). At 24 weeks, $71 \%$ of the patients receiving ETN plus MTX versus $27 \%$ of those receiving placebo plus MTX met the ACR20 criteria $(\mathrm{p}<0.001) ; 39 \%$ of the patients receiving ETN plus MTX and $3 \%$ of those receiving placebo plus MTX reached an ACR50 response $(p<0.001)$. Patients receiving both treatments had significantly better outcomes of all measures of disease activity and the only adverse events associated with ETN were mild injection-site reactions. No withdrawals occurred due to adverse events associated with ETN.

Etanercept was studied as monotherapy for patients with early RA. Bathon and colleagues (2000) studied 632 patients with early rheumatoid arthritis with either twice-weekly subcutaneous ETN (10 mg or $25 \mathrm{mg}$ ) or weekly oral MTX (mean dose $19 \mathrm{mg}$ per week) for 12 months. Although patients who received the $25 \mathrm{mg}$ dose of ETN had a more rapid rate of improvement, with significantly more patients achieving ACR 20\%, 50\%, and 70\% improvement in disease activity during the first six months $(\mathrm{p}<0.05)$ there was no difference between the groups in these outcome measures at 12 months. This was the first study to use rapidly escalated dosing of MTX, which, since then set the standard for its use in RA. However, rapid initial control of disease may explain the difference in erosion scores between the two groups. The mean increase in the erosion score during the first 6 months was 0.30 in the $25 \mathrm{mg}$ ETN group versus 0.68 
in the MTX group ( $p=0.001)$, and the respective increases during the first 12 months were 0.47 and $1.03(p=0.002)$. Among patients who received the $25 \mathrm{mg}$ twice weekly (biw) of ETN, $72 \%$ had no increase in the erosion score, versus $60 \%$ of patients in the MTX group $(p=0.007)$. The ETNtreated group of patients also had fewer adverse events $(p=0.02)$ and fewer infections $(p=0.006)$ than the group that was treated with MTX.

In none of the trials up to this point had ETN plus MTX been compared with both ETN alone and MTX alone, in MTX naïve patients. A three arm trial in established RA patients, who averaged 6 years of disease duration, was published by Klareskog and colleagues (2004) known as the TEMPO trial (Trial of Etanercept and Methotrexate with Radiographic Patient Outcomes). In this trial 682 patients with active rheumatoid arthritis were randomly treated with either ETN 25 mg (subcutaneously twice a week), oral MTX alone (up to $20 \mathrm{mg}$ every week), or the combination of MTX and ETN. Efficacy of the combination therapy was significantly greater at 24 weeks for the combination group compared with ETN alone or MTX alone. The ACR20 responses were respectively for the 3 groups $85 \%, 76 \%$, and $75 \%$ ); The ACR50 were at $69 \%, 48 \%$, and $43 \%$; finally the ACR70 were $43 \%, 24 \%$, and $19 \%$. Moreover, $48 \%$ of the patients in the combination group reached clinical remission as defined by the EULAR Disease Activity Score (DAS). The combination was also more efficacious than MTX or ETN alone in retardation of joint damage (mean total Sharp score $-0 \cdot 54$ [95\% confidence interval (CI) $-1 \cdot 00$ to -0.07$]$ vs $2.80[1.08$ to 4.51$], \mathrm{p}<0.0001$, and $0.52[-0.10$ to $1 \cdot 15$ ], $\mathrm{p}=0 \cdot 0006$; respectively). The number of patients reporting infections or adverse events was similar in all groups. The authors concluded that the combination of ETN and MTX was significantly better in reducing signs and symptoms of RA, improving functional disability, and retarding radiographic progression compared with MTX or ETN alone. Therefore, unless contraindicated, it is recommended to always combine ETN and MTX.

In daily clinical practice a great proportion of patients still have active disease despite adequate doses of MTX and hence require the addition of anti-TNF agents. The question arises whether MTX needs to be continued or can be safely stopped if treatment with ETN is initiated. This issue was addressed in the ADORE trial (Add Enbrel or Replace MTX) (Taggart et al 2005) which compared the efficacy and safety of ETN plus MTX with ETN alone in RA patients with an inadequate response to MTX therapy. Patients with active RA (DAS28 $\geq 3.2$ or $\geq 5$ swollen and painful joints and an ESR $\geq 10 \mathrm{~mm} / \mathrm{hr}$ ) despite treatment with MTX $\geq 12.5 \mathrm{mg} / \mathrm{wk}$ for $\geq 3$ months were randomly allocated to twice weekly therapy of ETN (25 mg biw) and MTX therapy or ETN $25 \mathrm{mg}$ biw alone. The monotherapy group of patients initially received ETN concurrently with MTX; however, the MTX was discontinued over a period of 4 weeks. A Health Assessment Questionnaire (HAQ) was administered at baseline and week 16 and EQ-5D and EQ$5 \mathrm{D}$ general health visual analog scale (EQ-5D GH VAS) at baseline, 4, 8, 12 and 16 weeks. Three hundred and fifteen patients were randomized to ETA $(n=160)$ or ETA-MTX $(n=155)$. At wk 16, both treatment groups demonstrated important improvements in all three quality of life assessments with no significant differences noted between the two groups. The proportion of patients in the ETA-MTX and ETA groups achieving improvements of HAQ $\geq 0.22$ (73.8\% vs $71.1 \%$, respectively) and HAQ $\geq 0.5$ (57.5\% vs $59.2 \%)$ as well as a normal HAQ of $\leq 0.5(32.6 \%$ vs $35.6 \%)$ were similar. The same percentage of patients in the ETAMTX and ETA groups attained a Global Health score at or above the population norms of $\geq 82$ (31.4\% vs $32.3 \%)$. Thus, for patients with side effects or intolerance to MTX switching treatment to ETN alone provides excellent therapeutic improvements in physical function and quality of life in the short term.

In a post hoc analysis, data from 4 trials were extracted and a combined analysis of safety and efficacy of ETN in patients who were $\geq 65$ years to those $<65$ years was completed (Flieschman et al 2003). All patients received ETN subcutaneously twice weekly. Of 1128 patients enrolled in ETN trials, 197 (17\%) were $\geq 65$ years of age. Clinical response, assessed by the ACR 20\%, 50\% and 70\% was rapid and sustained and did not differ between age groups. At one year, $69 \%$ of patients $<65$ years and $66 \%$ of patients $\geq 65$ years met the ACR20. Forty percent met the ACR50 and 17\% the ACR70. Although injection site reactions, headache, and rhinitis occurred somewhat more frequently in younger patients, the overall rates and types of other adverse events were comparable in both groups and ETN was well tolerated in the older age group.

Etanercept has most recently been shown to be effective when given as in a once weekly dosing regimen of $50 \mathrm{mg}$ subcutaneously (Keystone et al 2005). In this study evaluating pharmacokinetics, safety and efficacy of this new dosing strategy in comparison with $25 \mathrm{mg}$ SC biw $420 \mathrm{RA}$ patients were randomized to receive, in a blinded manner, the study drug for up to 16 weeks: 214 patients received $50 \mathrm{mg}$ ETN once weekly, 153 received $25 \mathrm{mg}$ ETN biw, and 
53 received placebo for 8 weeks followed by $25 \mathrm{mg}$ ETN biw for 8 weeks. Efficacy and safety were assessed at weeks 8 and 16 . The primary efficacy end point was achievement of the ACR 20 criteria at week 8 . It was achieved by $50 \%$ of the patients receiving $50 \mathrm{mg}$ ETN once weekly, by $49 \%$ of the patients receiving $25 \mathrm{mg}$ ETN biw, and by $19 \%$ of the patients in the placebo group ( $\mathrm{p} \leq 0.0001$ for each ETN group vs placebo). Similarly, achievement of the ACR50 response was attained by $18 \%$ of patients in each of the 2 ETN groups, compared with $6 \%$ of patients in the placebo group $(\mathrm{p}<0.03$ for each comparison). Pharmacokinetics of the 2 ETN regimens were similar at steady state. No clinically significant differences in efficacy or safety were observed between the 2 ETN groups.

The number needed to treat (NNT) is a helpful composite score for clinicians to help translate the results from clinical trials and systematic reviews. A low number needed to treat indicates that few patients would need to receive an agent to achieve a designated outcome. Recently Osiri and colleagues (2003) looked at the NNT from data in rheumatology clinical trials with ETN based on the ACR20 improvement. They noted that for RA, ETN treatment for six months had the smallest NNT $(1.6 ; 95 \%$ CI 1.4 to 2.0$)$.

\section{Etanercept and patients driven outcomes}

The impact of ETN on health-related quality of life (HRQOL) and functional status over time was analyzed in 533 RA patients who had been previously randomized in trials of ETN (Yelin et al 2001). Initial improvement in patient-centered outcomes including HAQ, physical component and mental component summary scores of the SF-36 were shown to be sustained over time in patients with either long term or early stage disease. The impact of ETN on healthcare use and employment was also evaluated in 260 patients with early RA from the Early Rheumatoid Arthritis (ERA) trial (Lubeck et al 2001). Even though the control group of patients who received ETN only at the termination of the ERA study received it for a mean of 239 days, patients originally randomized to ETN for a mean of 882 days reported fewer patient visits, outpatient surgeries, and hospital admissions for RA than controls. RA patients who were employed at disease onset and originally randomized to ETN had more hours of employment compared with controls. These data suggest the possibility of reducing healthcare utilization and increased employment with the use of ETN for a relatively short period of time.

\section{Efficacy of etanercept in psoriatic arthritis}

Etanercept has also been shown to be effective in psoriatic arthritis (PsA) (Mease et al 2000). In this 12 week randomized, double blind controlled trial, 60 patients with PsA and psoriasis were studied for efficacy and safety of ETN (25 mg biw SC) or placebo. The PsA outcome measures included the proportion of patients who met the Psoriatic Arthritis Response Criteria (PsARC) and those who met the ACR20 response criteria. Psoriasis endpoints included improvement in the Psoriasis Area and Severity Index (PASI) and improvement in prospectively identified individual target lesions. Eighty seven percent of ETNtreated patients met the PsARC compared with 23\% of placebo-controlled patients. The ACR20 was achieved by $73 \%$ of ETN-treated patients compared with $13 \%$ of placebo-treated patients. Of the 19 patients in each treatment group who could be assessed for psoriasis ( $\geq 3 \%$ body surface area), five $(26 \%)$ of ETN-treated patients achieved a $75 \%$ improvement in the PASI, compared with none of the placebo-treated patients $(p=0 \cdot 015)$. The median PASI improvement was $46 \%$ in ETN-treated patients versus $9 \%$ in placebo treated patients; similarly, median target lesion improvements were $50 \%$ and 0 , respectively.

\section{Safety of ETN in the rheumatoid arthritis trials and extension studies}

With the accumulated experience with the anti-TNF agents, certain areas of safety concerns have emerged, namely: serious infections, opportunistic infections including reactivation of tuberculosis (TB), malignancy, and more particularly lymphomas, autoimmune syndromes, demyelinating conditions, and congestive heart failure.

Through December 2002, 3839 patients (8336 patientyears exposure) were treated in clinical trials whereas 231000 patients (423000 patient-years exposure; all indications) were treated.

\section{Risk of infections with etanercept}

Given that TNF- $\alpha$ plays an important role in host defence against infection, inhibition of TNF- $\alpha$ is expected to increases the risk of infection in patients treated with TNF$\alpha$ antagonists (Cunnae et al 2003). However, in all of the ETN placebo-controlled double blind trials, no increase in the frequency or the nature of infections were noted, 
including serious infections, between ETN- and placebotreated patients. Moreover, in the early RA trial, serious infections were statistically higher in the MTX group compared with the ETN-treated patients. Because of the potential risk, it is recommended not to administer ETN to patients with recurrent infectious episodes and to stop this agent in the presence of infections requiring antibiotics. ETN may be restarted once the infection has resolved, although the decision to reinstate ETN therapy should be made on a case by case basis.

Several studies have evaluated the frequency of infections after ETN administration compared with the frequency prior to initiation. In one study, the rate of infection in 90 patients was compared with the rate of infection one year prior to therapy. The results showed a significant ( $\sim 2$ fold) increase in the incidence of recorded infections following the initiation of therapy for year 0 , for year 1, and for year 2 respectively (Belostocki et al 2001). Most infections were respiratory and the majority was not serious. Similar findings were observed in another study of 168 patients followed over one year (Philips et al 2002). In that study, nonserious infections were seen in $51 \%$ of patients during ETN therapy compared with $19 \%$ in the pretreatment period although the rate of serious infections was comparable ( $1.8 \%$ vs $2.9 \%)$ before and after therapy.

TNF has been shown to be critical to the maintenance of post-infectious granuloma generated in response to microbial agents such as Mycobacterium Tuberculosis $(\mathrm{MTb})$, histoplasmosis, and other opportunistic infections. From the post-marketing surveillance data available as of December 2001 for 114000 ETN-treated patients (150000 patient-years) world-wide, 20 cases of TB have been reported. The pattern of TB was consistent with significant immunosuppression with 3 of the patients exhibiting a miliary pattern. The median time of onset of reactivation was 6 months. Since the expected incidence of TB in the USA is $6-8 / 100000$ patient-years, it is unclear whether TB is increased in ETN-treated patients over the general RA population. Nevertheless, screening for latent TB with a chest $\mathrm{X}$-ray and Purified Protein Derivative Standard (PPD) skin test is recommended. A positive PPD $(\geq 5 \mathrm{~mm})$ is an indication for initiating therapy for latent TB using isoniazid (INH) (for 9 months) or rifampin (for 4 months). Other opportunistic infections have also been reported including atypical mycobacterium $(n=8)$, pneumocystis carini $(n=5)$, with a few cases of Candidiasis $(n=3)$, crypotococcosis $(n=3)$, aspergillosis $(n=2)$, and isolated cases of histoplasmosis $(\mathrm{n}=1)$, listeria monocytogenes $(\mathrm{n}=1)$.
Whether these numbers reflect an increase relative to conventional DMARDS is uncertain.

\section{Autoimmunity and lupus-like reactions}

In randomised control trials $11 \%$ of patients developed new ANAs compared with 5\% with placebo. With ETN 15\% developed anti-dsDNA antibodies compared with $4 \%$ of placebo-treated patients. A recent study demonstrated that prior to ETN 18\% (10/56) patients had positive anticardiolipin (ACA) and that after 6 months of treatment, $25 \%$ of patients tested positive. Increases were seen for both IgG and IgM ACA. Increasing age, a higher number of prior disease-modifying antirheumatic drugs and higher DAS28 were predictors of developing ACA (Jonsdotter et al 2004). Anti-thyroid and antineutrophil cytoplasmic antibodies were rarely found in the absence of a clinical picture indicative of thryroiditis or vasculitis respectively (De Rycke et al 2005).

Shakoor and colleagues (2002) described four cases of systemic lupus erythematosus (SLE)-like syndrome in patients treated with ETN. Symptoms appeared 6 weeks -3 months after initiation of ETN. Clinical presentation with the four cases included fever, malaise and arthritis; discoid lupus rash; hypertension, arthritis and erythematosus facial rash; and pleuritis and malar rash. Within 2-6 weeks, symptoms resolved with drug withdrawal. Mohan and colleagues (2002) used the FDA adverse event-reporting system to identify 16 cases of new-onset SLE after ETN use from November 1998 to February 2002, excluding Shakoor and colleagues' cases.

\section{Malignancy and lymphoma}

During the 2003 FDA review of safety with the TNF antagonists, 70 cases of lymphoma were reported on 230000 patients exposed to ETN. This would indicate the incidence of lymphoma is about 2 to 3 cases per 10000 patient-years and the estimated rate in the normal population of 3 per 10000 patient years (Wasko 2004). In the Swedish and other European registries the risk of lymphoma has also been shown to be increased in patients with RA (Askling et al 2005). Recently Wolfe and Michaud (2004a) examined the rate of standardized incidence ratio (SIR) and predictors for lymphoma in patients with RA and in RA patient subsets by treatment group in 18572 patients prospectively enrolled in the National Data Bank for Rheumatic Diseases (NDB) registry. Patients were surveyed biannually, and potential lymphoma cases received detailed follow up. Results were compared with the SEER (Survey, Epidemiology, and End 
Results) cancer database to derive the expected number of cases of lymphoma controlling for age and gender. The overall SIR for lymphoma was 1.9 (95\% CI 1.3-2.7). The SIR for biologic use was 2.9 (95\% CI 1.7-4.9) and for ETN, with or without infliximab (INF), the SIR was 3.8 (95\% CI 1.9-7.5) compared with 2.6 (95\% CI 1.4-4.5) for INF (with or without ETN). The SIR for MTX was 1.7 (95\% CI 0.93.2), and was 1.0 (95\% CI 0.4-2.5) for those not receiving MTX or biologics. Lymphoma was associated with higher age, male gender, and education. They concluded that lymphomas were increased in RA with the SIR being greatest for patients receiving anti-TNF therapies and that there were no statistical differences between ETA and INF therapies. The increased lymphoma rates observed with anti-TNF therapy was thought to reflect channeling bias, whereby patients with the highest risk of lymphoma (those with more severe disease) preferentially receive anti-TNF therapy and no causative relationship could be established on registry data. Very recently, a review of lymphoproliferative malignancies and solid tumors from 3 pharmacy databases did not show any increase in the patients treated with antiTNF therapies when compared with patients treated with MTX (Setogushi et al 2006)

\section{Demyelinating syndromes}

The number of cases of demyelination with ETN during the clinical trials is still unclear. Mohan and colleagues (2001) noted 17 cases of demyelination after ETN therapy. All neurological events were temporally related to the use of ETN with partial or complete resolution. The most common presenting clinical symptoms among the 20 patients were paresthesias (13 of 20) followed by visual disturbances secondary to optic neuritis ( 8 of 20). Other signs and symptoms included confusion (25\%), gait disturbance, apraxia, facial palsy, and Guillain-Barré syndrome. Four patients had multiple sclerosis.

Post-marketing observations did not show any increase beyond the expected numbers.

\section{Congestive heart failure and etanercept}

Results from two prospective randomized, double-blind trials comparing the effects of ETN and placebo in NYHA functional class II-IV CHF patients underwent a combined analysis (Mann et al 2004). The analysis showed no beneficial effect on death or congestive heart failure (CHF) hospitalization, with a relative risk of 1.11 (95\% CI 0.91 to $1.33, \mathrm{p}=0.33$ ) for ETN compared with placebo. For overall mortality, the estimated ETN-to-placebo relative risk was $1.13(95 \%$ CI 0.86 to $1.50, \mathrm{p}=0.39)$. Safety analysis of the trials showed a trend $(\mathrm{p}=0.067)$ toward more overall infections in the ETN-treated patients versus placebo in one of the trials, but not in the second one.

In a recent review from the NDB registry, Wolfe and Michaud (2004b) showed that there is a increased incidence of CHF among RA patients and that anti-TNF agents may improve this condition.

\section{Conclusion}

In summary, clinical trials show ETN is both safe and effective to use in RA and PsA. In RA, ETN has been shown not only to reduce disease activity but also to limit progression of joint damage in early and late disease. ETN can be used as a monotherapy or in combination with MTX, although the latter approach appears most effective in terms of reducing joint damage. Safety data in clinical trials have shown no significant differences compared with placebo. Thus the risk/benefit profile is manageable and ETN is a valuable treatment option for patients with RA and PsA.

\section{References}

Askling J, Fored CM, Baeckland E, et al. 2005. Haematopoietic malignancies in rheumatoid arthritis: lymphoma risk and characteristics after exposure to tumour necrosis factor antagonists. Ann Rheum Dis, 64:1414-20.

Bathon JM, Martin RW, Fleischmann RM, et al. 2000. Comparison of etanercept and methotrexate in patients with early rheumatoid arthritis. N Engl J Med, 343:1586-93.

Beayert R, Fiers W. 1998. Tumor necrosis factor and lymphotoxin. In: Mire-Sluis AR, Thorpe R (eds). Cytpkines 1st ed. London: Academic Pr, pp 235-60.

Belostocki KB, Leibowitz E, Tai K, et al. 2001. Infections associated with etanercept treatment in rheumatoid arthritis: 2 years of experience in the "real-world" [abstract]. Arthritis Rheum, 44(Suppl):S173, 725.

Berg L, Lampa J, van Vollenhoven R, et al. 2001. Increased peripheral T cell reactivity to microbial antigens and collagen type II in rheumatoid arthritis after treatment with soluble TNFa receptors. Ann Rheum Dis, 60:133-9.

Cartina AI, Lampa J, Ernestam S, et al. 2002. Anti tumor necrosis factor (TNF)-a therapy (etanercept) down regulates serum matrix metalloproteinase (MMP)-3 and MMP-1 in rheumatoid arthritis. Rheumatol, 41:484-9.

Cunnane G, Doran M, Bresnihan B. 2003. Infections and biological therapy in rheumatoid arthritis. Best Pract Res Clin Rheumatol, 17:345-63.

De Rycke L, Baeten D, Kruithof E, et al. 2005. Infliximab, but not etanercept, inducesIgM anti-double-stranded DNA autoantibodies as main antinuclear reactivity: biologic and clinical implications in autoimmune arthritis. Arthritis Rheum, 52:2192-201.

Fleischman RM, Baumgartner SW, Tindall EA, et al. 2003. Response to etanercept $\left(\right.$ Enbrel $\left.^{\circledR}\right)$ in elderly patients with rheumatoid arthritis: a retrospective analysis of clinical trial results. J Rheumatol, 30:691-6.

Jonsdotter T, Forshid J, Vollenhoven A, et al. 2004. Treatment with tumor necrosis factor antagonists in patients with rheumatoid arthritis induced anticardiolipin antibodies. Ann Rheum Dis, 63:1075-8. 
Keystone EC, Schiff MH, Kremer JM et al. 2005. Once-weekly administration of $50 \mathrm{mg}$ etanercept in patients with active rheumatoid arthritis: Results of a multicenter, randomized, double-blind, placebocontrolled trial. Arthritis Rheum, 50:353-63.

Klareskog L, van der Heijde D, de Jager JP, et al. 2004. Therapeutic effect of the combination of etanercept and methotrexate compared with each treatment alone in patients with rheumatoid arthritis: doubleblind randomised controlled trial. Lancet, 363:675-81.

Korth-Bradley JM, Rubin AS, Hanna RK, et al. 2000. The pharmacokinetics of etanercept in healthy volunteers. Ann Pharmacother, 34:161-4.

Krakauer T, Vilcek J, Oppenheim JJ. 1999. Proinflammatory cytokines: TNF and IL-1 families, chemokines, TGF-b, and others. In: Pauluis WE (ed). Fundamental immunology. 4th ed. Philadelphia: LippincottRaven, pp 775-811.

Locksley RM, Kileen N, Lenardo MJ. 2001. The TNF and TNF receptor superfamilies: integrating mammalian biology. Cell, 104:487-501.

Lubeck DP, Katz P, Yelin E et al. 2001. Long term impact of etanercept $\left(\right.$ Enbrel $\left.^{\circledR}\right)$ on health related quality of life and functional status of persons with rheumatoid arthritis [abstract]. Arthritis Rheum, 44(Suppl):S184, 787.

Mann DL, McMurray JJ, Packer M, et al. 2004. Targeted anticytokine therapy in patients with chronic heart failure: results of the Randomised Etanercept World-wide Evaluation (RENEWAL). Circulation, 109:1594-602.

McDermott MF. 2001. TNF and TNFR biology in health and disease. Cell Mol Biol, 47:619-35.

Mease PJ, Goffe BS, Metz J, et al. 2000. Etanercept in the treatment of psoriatic arthritis and psoriasis: a randomised trial. Lancet, 356:385-90.

Mohan AK, Edwards ET, Cote TR, et al. 2002. Drug induced systemic lupus and anti-TNFalpha blockers. Lancet, 360:646.

Mohan N, Edwards ET, Cupps TR, et al. 2001. Demyelination occurring during anti-tumor necrosis factor alpha therapy for inflammatory arthritides. Arthritis Rheum, 44:2862-9.

Mohler KM, Torrance DS, Smith CA, et al. 1993. Soluble tumor necrosis factor (TNF) receptors are effective therapeutic agents in lethal endotoxemia and function simultaneously as both TNF carriers and TNF antagonists. $J$ Immunol, 151:1548-61.

Moreland LW, Baumgartner SW, Schiff MH, et al. 1997. Treatment of rheumatoid arthritis with a recombinant human tumour necrosis factor receptor (p75)-Fc fusion protein, $N$ Engl J Med, 337:141-47.
Moreland LW, Bucy RP, Weinblatt ME, et al. 2002. Immune function in patients with rheumatoid arthritis treated with etanercept. Clin Immunol, 103:13-21.

Osiri M, Suarez-Almazor ME, Wells GA, et al. 2003. Number needed to treat (NNT): implication in rheumatology clinical practice. Ann Rheum Dis, 62:316-21.

Phillips K, Husni ME, Karlson EW, et al. 2002. Experience with etanercept in an academic medical center: Are infection rates increased? Arthritis Care Res, 47:17-21.

Setoguchi S, Solomon DH, Weinblatt ME, et al. 2006. Tumor Necrosis Factor a use and cancer in patients with rheumatoid arthritis. Arthritis Rheum, 54:2757-64.

Shakoor N, Michalski M, Harris CA, et al. 2002. Drug induced systemic lupus erythematosus associated with etanercept therapy. Lancet, 359:579-80.

Taggart AJ, van Riel PL, and Kekow J. 2005. Assessment of functional status and quality of life in a randomized study comparing etanercept and methotrexate with etanercept alone in patients with active rheumatoid arthritis despite methotrexate therapy: The ADORE study [abstract]. Arthritis Rheum, 52(Suppl):232, 550.

Verschueren PC, Markusse H, Smeets TJM, et al. 1999. Reduced cellularity and expression of adhesion molecules and cytokines after treatment with soluble human recombinant TNF receptor (p75) in RA patients [abstract]. Arthritis Rheum, 42(Suppl):S197, 762.

Wasko MC. 2004. Comorbid conditions in patients with rheumatic diseases: an update. Curr Opin Rheumatol, 16:109-13.

Weinblatt ME, Kremer JM, Bankhurst AD, et al. 1999. A trial of etanercept, a recombinant tumor necrosis factor receptor:fc fusion protein, in patients with rheumatoid arthritis receiving methotrexate. $N$ Engl $J$ Med, 340:253-9.

Wolfe F, Michaud K. 2004a. Lymphoma in rheumatoid arthritis: the effect of methotrexate and anti-tumor necrosis factor therapy in 18,572 patients. Arthritis Rheum, 50:1740-51.

Wolfe F, Michaud K. 2004b. Heart failure in rheumatoid arthritis: rates, predictors, and the effect of anti-tumor necrosis factor therapy. Am J Med, 116:305-11.

Yelin E, Katz P, Lubeck D et al. 2001. Impact of etanercept (Enbrel $\left.{ }^{\circledR}\right)$ on health care use and employment in early RA [abstract]. Arthritis Rheum, 44(Suppl):S152, 59520. 
